\title{
Modulation Format Independent Joint Polarization and Phase Tracking for Coherent Receivers
}

\author{
Cristian B. Czegledi, Erik Agrell, Senior Member, IEEE, Magnus Karlsson, Senior Member, IEEE, Fellow, OSA, \\ and Pontus Johannisson, Member, OSA
}

\begin{abstract}
The state of polarization and the carrier phase drift dynamically during transmission in a random fashion in coherent optical fiber communications. The typical digital signal processing solution to mitigate these impairments consists of two separate blocks that track each phenomenon independently. Such algorithms have been developed without taking into account mathematical models describing the impairments. We study a blind, model-based tracking algorithm to compensate for these impairments. The algorithm dynamically recovers the carrier phase and state of polarization jointly for an arbitrary modulation format. Simulation results show the effectiveness of the proposed algorithm, having a fast convergence rate and an excellent tolerance to phase noise and dynamic drift of the polarization at low complexity, which make the algorithm a strong candidate for future optical systems.
\end{abstract}

Index Terms-Coherent optical fiber communication, modelbased, phase noise, phase recovery, polarization demultiplexing, polarization drift, polarization recovery.

\section{INTRODUCTION}

D IGITAL signal processing (DSP) enables spectrally efficient communications based on coherent transmission. Contrary to traditional optical transmission links that are based on intensity-modulation and direct-detection, coherent transmissions carry the information in both the intensity and phase of the optical field, in both polarizations, and benefit from improved sensitivities, higher-order modulation formats, and digital impairment mitigation. Polarization-multiplexed quadrature phase-shift keying (PM-QPSK) introduced for 100 $\mathrm{Gb} / \mathrm{s}$ transmission has been widely deployed and reached maturity. Recently, $200 \mathrm{~Gb} / \mathrm{s}$ transceivers have been made commercially available based on 16-ary polarization-multiplexed quadrature amplitude modulation (PM-16-QAM) and it is expected that in the near future, higher-order PM- $M$-QAM modulation formats will become a necessity for higher data rates. However, the improved spectral efficiency comes at the cost of a reduced tolerance to impairments such as laser phase

C. B. Czegledi and E. Agrell are with the Department of Signals and Systems, Chalmers University of Technology, SE-412 96 Gothenburg, Sweden (e-mail: czegledi@chalmers.se, agrell@chalmers.se).

M. Karlsson and P. Johannisson are with the Department of $\mathrm{Mi}$ crotechnology and Nanoscience, Chalmers University of Technology, SE41296 Gothenburg, Sweden. (e-mail: magnus.karlsson@chalmers.se, pontus.johannisson@chalmers.se)

This work was supported by the Swedish research council (VR) and performed within the Fiber Optic Communications Research Center (FORCE) at Chalmers. The simulations were performed on resources at Chalmers Centre for Computational Science and Engineering (C3SE) provided by the Swedish National Infrastructure for Computing (SNIC).

Copyright (c) 2016 IEEE. noise and drift of the state of polarization (SOP), which have to be dynamically tracked in the receiver [1], [2].

The phase and SOP tracking are important DSP blocks at the receiver and are different from the chromatic dispersion compensation, which can be set once and then forgotten due to its static behavior. The SOP drift has its origin in the imperfections of the manufacturing process of the fiber cables, mechanical/thermal stress on the deployed fibers, splices, etc. Due to these random variations, the SOP changes dynamically in time and along the fiber, which makes it difficult to fully compensate for. The phase noise originates from the finite coherence length of the transmitter and receiver lasers and it drifts in time as a Wiener random walk. Despite the fact that the SOP drift and the phase noise arise due to different hardware imperfections, they can be modeled jointly as dynamic rotations of the optical field [3]. A deterministic or static behavior of these phenomena would be straightforward to resolve, but when the impairments drift randomly, the receiver must adjust dynamically to track the phenomena.

The common DSP solution for SOP tracking is done in the Jones space using the constant modulus algorithm (CMA) [2], initially developed for two-dimensional modulation formats [4], or modified versions of it to accommodate for various modulation formats, such as the multiple modulus algorithm (MMA) [5], [6] or the polarization-switched (PS-)CMA [7]. Alternatively, the polarization demultiplexing can be done in the Stokes space [8], [9], which in addition also aligns the phase of the two polarizations, thus improving the phase tracking by enabling joint phase estimation over the two polarizations. In general, the phase tracking is performed independently of the SOP tracking, using algorithms such as the Viterbi-Viterbi algorithm [10] or the blind phasesearch algorithm (BPS) [11], which treat each polarization independently.

Recently, Louchet et al. proposed the Kabsch algorithm [12], which addresses the two impairments jointly in both polarizations in the real four-dimensional (4D) space and accommodates any modulation format. In general, joint estimation leads to better performance, and it is expected that future transceivers will benefit from improved performance from such integrations of different DSP blocks [13].

However, very few algorithms present in the literature take into consideration analytical models describing the behavior of the impairments. Model-based algorithms have a restricted flexibility and therefore fewer degrees of freedom (DOFs) to adjust. The DOFs of model-based algorithms are restricted to only the ones covered by the impairment to compensate 
for, thus resulting in a more efficient impairment cancellation, rather than scanning over a larger domain in order to find the optimal setup.

In this paper, we propose a model-based algorithm to jointly recover the carrier phase and SOP for arbitrary modulation formats. The design of the algorithm is based on a channel model (described in Section II) that can emulate temporal stochastic polarization and phase drifts, and has been successfully validated with data measured on installed fibers [3]. The algorithm (described in Section III) uses a nondata aided decision-directed architecture, hence zero overhead, and operates jointly on both polarizations. The performance of the algorithm is investigated in Section IV by comparing it with state-of-the-art algorithms for different modulation formats, whereas the complexity is evaluated in Section V. The proposed algorithm performs similarly or better than stateof-the-art algorithms and provides a good trade-off between complexity and performance regardless of the modulation format. High performance and fast convergence rate at low complexity, for any modulation format, make the algorithm a strong candidate for future elastic optical systems, where the modulation format can be changed dynamically during transmission to accommodate for various channel and network conditions.

The following notation conventions are used throughout the paper: column vectors are denoted by bold lower case (e.g., u) and matrices by bold upper case (e.g., U), except a few specific cases, for literature consistency reasons, denoted by small Greek letters such as the Pauli matrices $\sigma_{i}$, the 4D basis matrices $\boldsymbol{\rho}_{i}, \boldsymbol{\lambda}_{i}$, and the electric field Jones vector $\mathbf{E}$. Transposition is written as $\mathbf{u}^{\mathrm{T}}$, conjugation as $\mathbf{u}^{*}$, and conjugate transpose as $\mathbf{u}^{\mathrm{H}}$. The $n \times n$ identity matrix is written as $\mathbf{I}_{n}$ and the expectation operator as $\mathbb{E}[\cdot]$. The dot operation $\boldsymbol{\alpha} \cdot \overrightarrow{\boldsymbol{\sigma}}$ should be interpreted as a linear combination of the three matrices forming the tensor $\vec{\sigma}=\left(\sigma_{1}, \sigma_{2}, \sigma_{3}\right)$. Multiplication of a matrix with the tensor $\vec{\sigma}$ result in a tensor with element-wise multiplications, e.g., $\mathbf{U} \overrightarrow{\boldsymbol{\sigma}}=\left(\mathbf{U} \boldsymbol{\sigma}_{1}, \mathbf{U} \boldsymbol{\sigma}_{2}, \mathbf{U} \boldsymbol{\sigma}_{3}\right)$. The absolute value is denoted by $|\cdot|$ and the Euclidean norm by $\|\cdot\|$.

\section{Discrete-Time Channel Model}

The coherent optical signal has two quadratures in two polarizations and can be described by a Jones vector

$$
\mathbf{E}(z, t)=\left(\begin{array}{l}
E_{x}(z, t) \\
E_{y}(z, t)
\end{array}\right)
$$

at propagation distance $z$ and time $t$, where $E_{x}\left(E_{y}\right)$ is the $x$ polarized ( $y$-polarized) electric field, represented as a complex baseband signal. The linearly modulated transmitted electric field into the transmission medium is

$$
\mathbf{E}(0, t)=\sum_{k} \mathbf{u}_{k} p(t-k T),
$$

where $\mathbf{u}_{k} \in \mathbb{C}^{2}$ are the information symbols for $k \in \mathbb{Z}, T$ is the symbol (baud) interval, and $p(t)$ is a real-valued pulse shape.
The received discrete symbols, at distance $L$, are obtained from the received electric field $\mathbf{E}(L, t)$ after matched filtering and sampling

$$
\mathbf{r}_{k}=\int_{-\infty}^{\infty} \mathbf{E}(L, t) p^{*}(t-k T) \mathrm{d} t
$$

The discrete transmitted symbols $\mathbf{u}_{k}$ are drawn independently from a finite constellation $\mathscr{C}=\left\{\mathbf{c}_{1}, \mathbf{c}_{2}, \ldots, \mathbf{c}_{M}\right\}$ with equal probability. The average energy per symbol is the average of $\left\|\mathbf{u}_{k}\right\|^{2}$ and in this case equals

$$
E_{s}=\frac{1}{M} \sum_{k=1}^{M}\left\|\mathbf{c}_{k}\right\|^{2}
$$

Assuming that the chromatic dispersion has been successfully compensated for and polarization-dependent losses and polarization mode dispersion are negligible, the propagation of the optical field can be described by a unitary $2 \times 2$ complexvalued Jones matrix $\mathbf{J}_{k}[14$, p. 18]. The received symbol $\mathbf{r}_{k} \in \mathbb{C}^{2}$, in the presence of optical amplifier noise, SOP drift, and laser phase noise, can be related to the input $\mathbf{u}_{k}$ as

$$
\mathbf{r}_{k}=e^{-i \phi_{k}} \mathbf{J}_{k} \mathbf{u}_{k}+\mathbf{n}_{k}
$$

where $i=\sqrt{-1}, \phi_{k}$ models the carrier phase noise, and $\mathbf{n}_{k} \in \mathbb{C}^{2}$ denotes the additive noise, which is represented by two independent complex circular zero-mean Gaussian random variables with variance $N_{0} / 2$ per real dimension, i.e., $\mathbb{E}\left[\mathbf{n}_{k} \mathbf{n}_{k}^{\mathrm{H}}\right]=N_{0} \mathbf{I}_{2}[15]$.

The phase noise is modeled as a Wiener process [11], [16]

$$
\phi_{k+1}=\dot{\phi}_{k}+\phi_{k}
$$

where $\dot{\phi}_{k}$ is the innovation of the phase noise. The innovation $\dot{\phi}_{k}$ is a random variable drawn independently at each time instance $k$ from a zero-mean Gaussian distribution

$$
\dot{\phi}_{k} \sim \mathcal{N}\left(0, \sigma_{\nu}^{2}\right)
$$

where the variance $\sigma_{\nu}^{2}=2 \pi \Delta \nu T, \Delta \nu$ is the sum of the linewidths of the transmitter and receiver lasers. The initial phase $\phi_{0}$ is modeled as a random variable uniformly distributed in the interval $[0,2 \pi)$.

The time evolution of the SOP drift can be emulated by modeling $\mathbf{J}_{k}$ as a sequence of random Jones matrices [3]

$$
\mathbf{J}_{k+1}=J\left(\dot{\boldsymbol{\alpha}}_{k}\right) \mathbf{J}_{k}
$$

where $J\left(\dot{\boldsymbol{\alpha}}_{k}\right)$ is the SOP innovation matrix (cf. $\dot{\phi}_{k}$ in (6)). The matrix function $J(\boldsymbol{\alpha})$ is defined using the matrix exponential [17, p. 165] parameterized by three DOFs $\boldsymbol{\alpha}[18]$ as

$$
J(\boldsymbol{\alpha})=\exp (-i \boldsymbol{\alpha} \cdot \overrightarrow{\boldsymbol{\sigma}}),
$$

where $\boldsymbol{\alpha}=\left(\alpha_{1}, \alpha_{2}, \alpha_{3}\right)$ is a three-component real vector and $\overrightarrow{\boldsymbol{\sigma}}=\left(\boldsymbol{\sigma}_{1}, \boldsymbol{\sigma}_{2}, \boldsymbol{\sigma}_{3}\right)$ is a tensor of the Pauli spin matrices [14, eq. (2.5.19)]

$$
\boldsymbol{\sigma}_{1}=\left(\begin{array}{cc}
1 & 0 \\
0 & -1
\end{array}\right), \sigma_{2}=\left(\begin{array}{ll}
0 & 1 \\
1 & 0
\end{array}\right), \quad \sigma_{3}=\left(\begin{array}{cc}
0 & -i \\
i & 0
\end{array}\right) .
$$

The vector $\boldsymbol{\alpha}$ can be expressed as a product $\boldsymbol{\alpha}=\theta \mathbf{a}$ of its length $\theta=\|\boldsymbol{\alpha}\|$ and the unit vector $\mathbf{a}=\left(a_{1}, a_{2}, a_{3}\right)$, which 


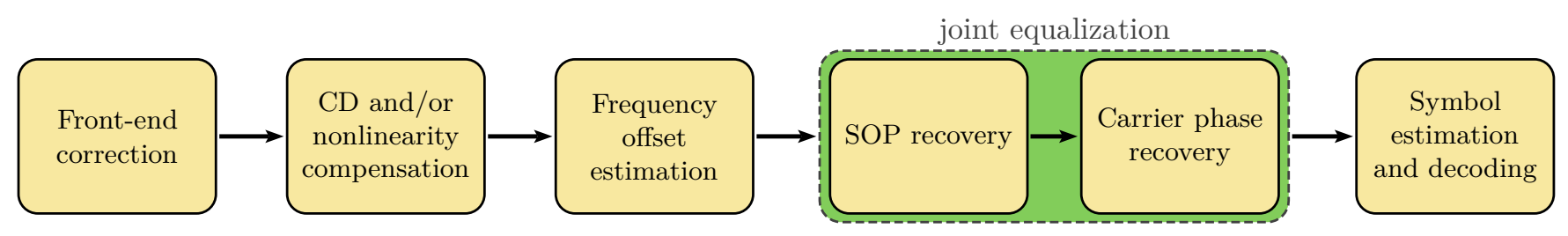

Figure 1. Receiver block diagram with elementary DSP modules.

represents its direction on the unit sphere. Based on this decomposition of $\boldsymbol{\alpha}$, (9) can be rewritten into an explicit form

$$
J(\boldsymbol{\alpha})=\mathbf{I}_{2} \cos \theta-i \mathbf{a} \cdot \overrightarrow{\boldsymbol{\sigma}} \sin \theta .
$$

Since the transformation $J(\boldsymbol{\alpha})$ is unitary, the inverse can be found by the conjugate transpose operation or negating the argument, $J(\boldsymbol{\alpha})^{-1}=J(\boldsymbol{\alpha})^{\mathrm{H}}=J(-\boldsymbol{\alpha})$.

The random nature of the SOP drift is emulated by drawing the three innovation parameters $\dot{\boldsymbol{\alpha}}_{k}$ of the innovation $J\left(\dot{\boldsymbol{\alpha}}_{k}\right)$ independently from a zero-mean real Gaussian distribution at each time instance $k$

$$
\dot{\boldsymbol{\alpha}}_{k} \sim \mathcal{N}\left(\mathbf{0}, \sigma_{p}^{2} \mathbf{I}_{3}\right),
$$

where $\sigma_{p}^{2}=2 \pi \Delta p T$ and $\Delta p$ is referred as the polarization linewidth, which quantifies the speed of the SOP drift, analogously to the linewidth describing the phase noise, cf. (7).

The initial state of the channel $\mathbf{J}_{0}=J\left(\boldsymbol{\alpha}_{0}\right)$ is formed from the vector $\alpha_{0}=\theta \mathbf{a}$, which is identified from the unit vector $\left(\cos \theta, a_{1} \sin \theta, a_{2} \sin \theta, a_{3} \sin \theta\right)^{\mathrm{T}}=\mathbf{g} /\|\mathbf{g}\|$ where $\mathbf{g} \sim \mathcal{N}\left(\mathbf{0}, \mathbf{I}_{4}\right)$. This ensures that $\mathbf{J}_{0} \mathbf{u}$ is uniformly distributed over all possible SOP for a fixed $\mathbf{u}$ [3], [19].

The phase noise and the SOP drift can be combined into a single operation $\mathbf{H}_{k}=e^{-i \phi_{k}} \mathbf{J}_{k}$ and (5) can be rewritten as

$$
\mathbf{r}_{k}=\mathbf{H}_{k} \mathbf{u}_{k}+\mathbf{n}_{k} \text {. }
$$

The update of $\mathbf{H}_{k}$ can be expressed analogously to (8) as

$$
\mathbf{H}_{k+1}=H\left(\dot{\phi}_{k}, \dot{\boldsymbol{\alpha}}_{k}\right) \mathbf{H}_{k},
$$

where the phase innovation $\dot{\phi}_{k}$ and the random vector $\dot{\boldsymbol{\alpha}}_{k}$ are defined as (7) and (12), respectively. The matrix function $H(\phi, \boldsymbol{\alpha})$ can be expressed as

$$
\begin{aligned}
H(\phi, \boldsymbol{\alpha}) & =e^{-i \phi} J(\boldsymbol{\alpha})=\exp \left(-i\left(\boldsymbol{\alpha} \cdot \overrightarrow{\boldsymbol{\sigma}}+\phi \mathbf{I}_{2}\right)\right) \\
& =(\cos \phi-i \sin \phi)\left(\mathbf{I}_{2} \cos \theta-i \mathbf{a} \cdot \overrightarrow{\boldsymbol{\sigma}} \sin \theta\right),
\end{aligned}
$$

which combines the effects of both phase noise and SOP drift.

\section{Polarization And Phase Tracking Algorithm}

In order to successfully decode the data at the receiver, the channel matrix $\mathbf{H}_{k}$ (or, equivalently, $\phi_{k}$ and $\mathbf{J}_{k}$ ) needs to be estimated and tracked during transmission such that it is possible to accurately estimate $\mathbf{u}_{k}$ from the received sample $\mathbf{r}_{k}$. This section provides a description of the proposed algorithm to estimate $\mathbf{H}_{k}$, first using the Jones formalism, thereafter alternatives using the Stokes and real 4D formalisms are given.

The Jones description can be replaced by the Stokes or real 4D descriptions, which can provide benefits in some situations [3]. The analytics describing wave propagation based on the Jones formalism rely on complex two-dimensional vectors and matrices that have four DOFs. This description is sufficient for wave propagation since it can cover any linear phenomenon that can arise during photon propagation. The Stokes description is preferred in some situations since the Stokes vectors are observable quantities and can be visualized as points on a three-dimensional sphere, called the Poincaré sphere. In this case, the channel matrix $\mathbf{J}_{k}$ is replaced by a $3 \times 3$ Mueller matrix $\mathbf{M}_{k}$ with three DOFs that models only the changes of the SOP. The Stokes description cannot model absolute phase shifts, therefore it is immune to phase noise. The real $4 \mathrm{D}$ formalism models the channel behavior using $4 \times 4$ real rotation matrices that have six DOFs, which span over a richer space than the Jones (four DOFs) or Mueller (three DOFs) matrices can. However, only four of them are physically realizable for propagating photons and the other two can be synthesized using DSP [20].

In the remainder of this section, we will provide the derivation of the proposed tracking algorithm using the Jones description, after which the equivalent algorithms using the Stokes and the real 4D descriptions are given. The details of the channel model descriptions using the Stokes and real 4D descriptions are omitted and can be found in [3].

\section{A. Jones Description}

The considered DSP setup is shown in Fig. 1, where we combine the SOP drift and carrier phase tracking, i.e., estimation of $\mathbf{H}_{k}$, into a single block after the frequency offset compensation, which can be done in this case using spectrum-based methods [21], [22]. Considering that $\mathbf{H}_{k}$ does not change significantly over a symbol duration, we estimate the transmitted symbol from $\hat{\mathbf{H}}_{k}^{-1} \mathbf{r}_{k}$ based on a previously calculated estimate of the channel matrix $\hat{\mathbf{H}}_{k}$ using the minimum Euclidean distance criterion

$$
\hat{\mathbf{u}}_{k}=\underset{\mathbf{c} \in \mathscr{C}}{\arg \min }\left\|\hat{\mathbf{H}}_{k}^{-1} \mathbf{r}_{k}-\mathbf{c}\right\|^{2} .
$$

Thereafter $\hat{\mathbf{H}}_{k}$ is updated as

$$
\hat{\mathbf{H}}_{k+1}=H\left(\hat{\phi}_{k}, \hat{\boldsymbol{\alpha}}_{k}\right) \hat{\mathbf{H}}_{k},
$$

where $\hat{\phi}_{k}$ and $\hat{\boldsymbol{\alpha}}_{k}$ are estimates of $\dot{\phi}_{k}$ and $\dot{\boldsymbol{\alpha}}_{k}$. These estimates are calculated such that the error function

$$
e_{k}=\left\|\left(H(\phi, \boldsymbol{\alpha}) \hat{\mathbf{H}}_{k}\right)^{-1} \mathbf{r}_{k}-\hat{\mathbf{u}}_{k}\right\|^{2},
$$

is minimized with respect to $\phi$ and $\alpha$, i.e.,

$$
\left[\hat{\phi}_{k}, \hat{\boldsymbol{\alpha}}_{k}\right]=\underset{\phi, \boldsymbol{\alpha}}{\arg \min } e_{k} .
$$


This can be achieved by computing $\hat{\phi}_{k}$ and $\hat{\boldsymbol{\alpha}}_{k}$ using the gradient descent method [23, p. 466]

$$
\begin{aligned}
& \hat{\phi}_{k}=-\mu_{\phi} \operatorname{Re}\left(\left.\frac{\partial e_{k}}{\partial \phi}\right|_{\substack{\phi=0 \\
\boldsymbol{\alpha}=[0,0,0]^{\mathrm{T}}}}\right) \\
& =-2 \mu_{\phi} \operatorname{Re}\left(i\left(\hat{\mathbf{H}}_{k}^{-1} \mathbf{r}_{k}-\hat{\mathbf{u}}_{k}\right)^{\mathrm{H}} \hat{\mathbf{H}}_{k}^{-1} \mathbf{r}_{k}\right) \text {, } \\
& \hat{\boldsymbol{\alpha}}_{k}=-\mu_{\boldsymbol{\alpha}} \operatorname{Re}\left(\left.\nabla_{\boldsymbol{\alpha}} e_{k}\right|_{\substack{\phi=0 \\
\boldsymbol{\alpha}=[0,0,0]^{\mathrm{T}}}}\right) \\
& =-2 \mu_{\boldsymbol{\alpha}} \operatorname{Re}\left(i\left(\hat{\mathbf{H}}_{k}^{-1} \mathbf{r}_{k}-\hat{\mathbf{u}}_{k}\right)^{\mathrm{H}} \hat{\mathbf{H}}_{k}^{-1} \overrightarrow{\boldsymbol{\sigma}} \mathbf{r}_{k}\right),
\end{aligned}
$$

where $\mu_{\phi}$ and $\mu_{\boldsymbol{\alpha}}$ are positive tracking step sizes of the phase and of the SOP parameters, respectively, which determine the speed of the algorithm's convergence, the tracking accuracy, and the rate at which changes in the channel can be tracked. The derivations of (22) and (24) can be found in the Appendix. Both innovation parameters $\dot{\phi}_{k}$ and $\dot{\boldsymbol{\alpha}}_{k}$ have zero mean by (7) and (12); therefore the partial derivatives in (21) and (23) are evaluated at $\phi=0, \alpha=[0,0,0]^{\mathrm{T}}$, which results in no preferred direction of $\hat{\phi}_{k}$ and $\hat{\boldsymbol{\alpha}}_{k}$. Evaluating the gradient at non-zero values could compensate for constant offsets; e.g., using $\phi \neq 0$ could compensate for frequency offsets.

It is important to note that $H(\phi, \boldsymbol{\alpha})$ is a many-to-one function. Therefore $\hat{\phi}_{k}$ and $\hat{\boldsymbol{\alpha}}_{k}$ are not necessarily equal to $\dot{\phi}_{k}, \dot{\boldsymbol{\alpha}}_{k}$ and can have different values, but resulting in same matrix $H(\phi, \boldsymbol{\alpha})$.

Since the typical drift time of the SOP is slower $(\sim 1 \mathrm{~ms}$ or larger) [3], [24], [25] than the drift of the phase noise ( $1 \mu \mathrm{s})$ [11], we chose the tracking steps $\mu_{\phi}, \mu_{\boldsymbol{\alpha}}$ of the two phenomena differently. For the same reasons, the update of the SOP estimate $\hat{\boldsymbol{\alpha}}_{k}$ can be done less often than the update of the absolute phase estimate $\hat{\phi}_{k}$, which will result in a decreased DSP complexity. In this case, $\hat{\phi}_{k}$ can be calculated using (22) at every time instance $k$ and $\hat{\boldsymbol{\alpha}}_{k}$ using (24) only at every $P$ symbols, otherwise should be set to $[0,0,0]^{\mathrm{T}}$.

Fig. 2 shows an example of the algorithm's tracking capability, where we compare the sum of the innovations $\dot{\phi}_{k}, \dot{\boldsymbol{\alpha}}_{k}$ with their estimates $\hat{\phi}_{k}, \hat{\boldsymbol{\alpha}}_{k}$ obtained using the proposed algorithm. Even though, as mentioned above, $\hat{\phi}_{k}, \hat{\boldsymbol{\alpha}}_{k}$ do not have to follow $\dot{\phi}_{k}, \dot{\boldsymbol{\alpha}}_{k}$ to obtain a good estimate of the channel matrix $\mathbf{H}_{k}$, the algorithm manages to obtain similar parameters efficiently without exhibiting cycle slips over the $10^{5}$ simulated symbols. Perhaps different values of $\hat{\phi}_{k}, \hat{\boldsymbol{\alpha}}_{k}$ may be obtained if the initial values $\hat{\phi}_{0}, \hat{\boldsymbol{\alpha}}_{0}$ are not set to be the same as $\phi_{0}, \boldsymbol{\alpha}_{0}$. Note that the plotted parameters are just for demonstration purposes and do not reflect the behavior of $\mathbf{H}_{k}$ or $\hat{\mathbf{H}}_{k}$, since $\mathbf{H}_{k} \neq H\left(\sum_{k} \dot{\phi}_{k}, \sum_{k} \dot{\boldsymbol{\alpha}}_{k}\right)$ because in general $H\left(\phi_{1}+\phi_{2}, \boldsymbol{\alpha}_{1}+\boldsymbol{\alpha}_{2}\right) \neq H\left(\phi_{1}, \boldsymbol{\alpha}_{1}\right) H\left(\phi_{2}, \boldsymbol{\alpha}_{2}\right)$.

In a prestudy for this work [26], we investigated a similar algorithm that tracks both the carrier phase and the SOP jointly. That algorithm was based on the assumption that $\alpha_{1}$, $\alpha_{2}$, and $\alpha_{3}$ corresponding to $\mathbf{J}_{k}$ drift as independent Wiener processes, in analogy with $\phi$. While intuitively reasonable, this assumption turned out on closer investigation to be physically incorrect, and the algorithm in [26] is therefore not in its original form suitable for implementation in fiber-optical

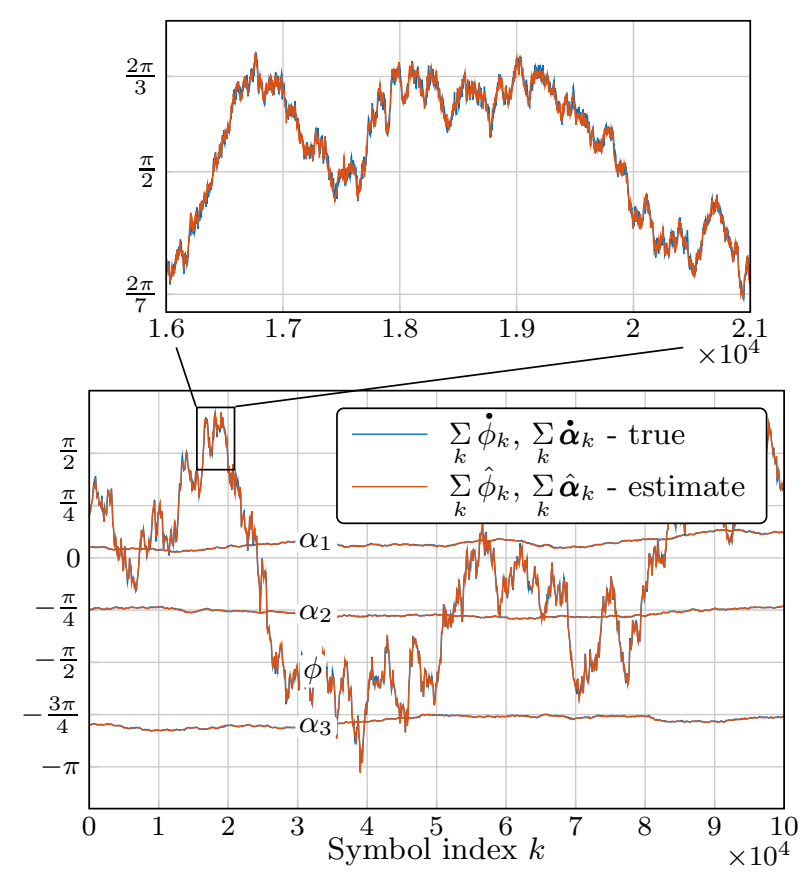

Figure 2. Tracked channel parameters using the proposed algorithm with $E_{s} / N_{0}=21.28 \mathrm{~dB}, \Delta \nu=1 \mathrm{MHz}$, and $\Delta p=1 \mathrm{kHz}$ at 28 Gbaud PM16-QAM are shown. As can be seen, the algorithm has excellent tracking capabilities without exhibiting cycle slips.

transmission systems. In contrast, the work in this paper is derived from a physically more accurate SOP drift model, which is based on matrix multiplication (8) and was proposed and validated in [3].

\section{B. Stokes Description}

In this case, the propagation of the electric field can be modeled as [3]

$$
\mathbf{s}_{\mathbf{r}_{k}}=\mathbf{M}_{k} \mathbf{s}_{\mathbf{u}_{k}}+\mathbf{s}_{\mathbf{n}_{k}}
$$

where $\mathbf{s}_{\mathbf{u}_{k}}=\mathbf{u}_{k}^{\mathrm{H}} \overrightarrow{\boldsymbol{\sigma}} \mathbf{u}_{k}$ and $\mathbf{s}_{\mathbf{r}_{k}}=\mathbf{r}_{k}^{\mathrm{H}} \overrightarrow{\boldsymbol{\sigma}} \mathbf{r}_{k}$ are the corresponding Stokes vectors of $\mathbf{u}_{k}$ and $\mathbf{r}_{k}$ [14, eq. (2.5.26)]. The noise term is $\mathbf{s}_{\mathbf{n}_{k}}=\left(\mathbf{H}_{k} \mathbf{u}_{k}\right)^{\mathrm{H}} \overrightarrow{\boldsymbol{\sigma}} \mathbf{n}_{k}+\mathbf{n}_{k}^{\mathrm{H}} \overrightarrow{\boldsymbol{\sigma}} \mathbf{H}_{k} \mathbf{u}_{k}+\mathbf{n}_{k}^{\mathrm{H}} \overrightarrow{\boldsymbol{\sigma}} \mathbf{n}_{k}$ and can be identified by equating terms after substituting (13) in $\mathbf{s}_{\mathbf{r}_{k}}=\mathbf{r}_{k}^{\mathrm{H}} \overrightarrow{\boldsymbol{\sigma}} \mathbf{r}_{k}$. As can be noted, $\mathbf{s}_{\mathbf{n}_{k}}$ is signal dependent and (31) is not an additive noise model, opposed to (13). The channel matrix $\mathbf{M}_{k}$ modeling the evolution of the SOP can be expressed using a $3 \times 3$ Mueller matrix defined as [18]

$$
M(\boldsymbol{\alpha})=\exp (2 \mathcal{K}(\boldsymbol{\alpha})),
$$

where $\mathcal{K}(\boldsymbol{\alpha})$ denotes the cross-product operator [20, eq. (11)]

$$
\mathcal{K}(\boldsymbol{\alpha})=\left(\begin{array}{ccc}
0 & -\boldsymbol{\alpha}_{3} & \boldsymbol{\alpha}_{2} \\
\boldsymbol{\alpha}_{3} & 0 & -\boldsymbol{\alpha}_{1} \\
-\boldsymbol{\alpha}_{2} & \boldsymbol{\alpha}_{1} & 0
\end{array}\right)
$$

The inverse can be obtained as $M(\boldsymbol{\alpha})^{-1}=M(\boldsymbol{\alpha})^{\mathrm{T}}=$ $M(-\boldsymbol{\alpha})$. The polarization transformations introduced by $\mathbf{M}_{k}$ can be seen as rotations of the Poincaré sphere around the unit vector a by an angle $2 \theta$. 
Analogously to the Jones description, the algorithm decides first which was the transmitted Stokes vector based on the minimum Euclidean distance criterion

$$
\hat{\mathbf{s}}_{\mathbf{u}_{k}}=\underset{\mathbf{c} \in \mathscr{C}}{\arg \min }\left\|\hat{\mathbf{M}}_{k}^{-1} \mathbf{s}_{\mathbf{r}_{k}}-\mathbf{s}_{\mathbf{c}}\right\|^{2}
$$

using the inverted estimate of $\mathbf{M}_{k}$, where ${ }^{1} \mathbf{s}_{\mathbf{c}}=\mathbf{c}^{\mathrm{H}} \overrightarrow{\boldsymbol{\sigma}} \mathbf{c}$. Thereafter, $\hat{\mathbf{M}}_{k}$ is updated analogously to (18) as

$$
\hat{\mathbf{M}}_{k+1}=M\left(\hat{\boldsymbol{\alpha}}_{k}\right) \hat{\mathbf{M}}_{k} \text {. }
$$

Analogously with (20)-(24), it can be shown that the optimal $\hat{\boldsymbol{\alpha}}_{k}=\left[\hat{\alpha}_{k, 1}, \hat{\alpha}_{k, 2}, \hat{\alpha}_{k, 3}\right]^{\mathrm{T}}$ are computed as

$$
\hat{\alpha}_{k, i}=4 \mu_{\boldsymbol{\alpha}}\left(\hat{\mathbf{M}}_{k}^{-1} \mathbf{s}_{\mathbf{r}_{k}}-\hat{\mathbf{s}}_{\mathbf{u}_{k}}\right)^{\mathrm{T}} \hat{\mathbf{M}}_{k}^{-1} \mathcal{K}\left(\mathbf{e}_{i}\right) \mathbf{s}_{\mathbf{r}_{k}}
$$

for $i=1,2,3$, using the gradient descent algorithm such that the Euclidean distance in the Stokes space is minimized. The vectors $\mathbf{e}_{i}$ form the standard basis in $\mathbb{R}^{3}$.

Note that the error function that minimizes the Euclidean distance is not optimum in this case since $\mathbf{s}_{\mathbf{n}_{k}}$ includes not only noise, but signal-noise interaction. Furthermore, the noise term $\mathbf{n}_{k}^{\mathrm{H}} \overrightarrow{\boldsymbol{\sigma}} \mathbf{n}_{k}$ is non-Gaussian. Better metrics [8] that take into account the non-Gaussian distribution of the noise can be used, but it is outside the scope of this work.

\section{4D Real Description}

In the 4D formalism, the phase and SOP drifts are combined and modeled using a $4 \times 4$ real orthogonal matrix $\mathbf{R}_{k}$ [20], [27], [28] as

$$
\mathbf{v}_{\mathbf{r}_{k}}=\mathbf{R}_{k} \mathbf{v}_{\mathbf{u}_{k}}+\mathbf{v}_{\mathbf{n}_{k}}
$$

where $\mathbf{v}_{\mathbf{z}}$ for any $\mathbf{z}=\left[z_{1}, z_{2}\right]^{\mathrm{T}} \in \mathbb{C}^{2}$ is defined as $\left[\operatorname{Re}\left(z_{1}\right), \operatorname{Im}\left(z_{1}\right), \operatorname{Re}\left(z_{2}\right), \operatorname{Im}\left(z_{2}\right)\right]^{\mathrm{T}}$. The channel matrix $\mathbf{R}_{k}$ can be expressed using the matrix function [20]

$$
R(\phi, \boldsymbol{\alpha})=\exp ((\phi, 0,0) \cdot \overrightarrow{\boldsymbol{\lambda}}-\boldsymbol{\alpha} \cdot \overrightarrow{\boldsymbol{\rho}}),
$$

where $\overrightarrow{\boldsymbol{\rho}}=\left(\boldsymbol{\rho}_{1}, \boldsymbol{\rho}_{2}, \boldsymbol{\rho}_{3}\right)$ and $\vec{\lambda}=\left(\boldsymbol{\lambda}_{1}, \boldsymbol{\lambda}_{2}, \boldsymbol{\lambda}_{3}\right)$ are six ${ }^{2}$ constant basis matrices [20, eqs. (20)-(25)]

$$
\begin{aligned}
\boldsymbol{\rho}_{1} & =\left(\begin{array}{cccc}
0 & -1 & 0 & 0 \\
1 & 0 & 0 & 0 \\
0 & 0 & 0 & 1 \\
0 & 0 & -1 & 0
\end{array}\right), \boldsymbol{\rho}_{2}=\left(\begin{array}{cccc}
0 & 0 & 0 & -1 \\
0 & 0 & 1 & 0 \\
0 & -1 & 0 & 0 \\
1 & 0 & 0 & 0
\end{array}\right), \\
\boldsymbol{\rho}_{3} & =\left(\begin{array}{cccc}
0 & 0 & 1 & 0 \\
0 & 0 & 0 & 1 \\
-1 & 0 & 0 & 0 \\
0 & -1 & 0 & 0
\end{array}\right), \lambda_{1}=\left(\begin{array}{cccc}
0 & 1 & 0 & 0 \\
-1 & 0 & 0 & 0 \\
0 & 0 & 0 & 1 \\
0 & 0 & -1 & 0
\end{array}\right) .
\end{aligned}
$$

The inverse can be obtained as $R(\phi, \boldsymbol{\alpha})^{-1}=R(\phi, \boldsymbol{\alpha})^{\mathrm{T}}=$ $R(-\phi,-\boldsymbol{\alpha})$.

Analogously to the Jones/Stokes description, the algorithm decides first which was the transmitted symbol based on the minimum Euclidean distance criterion

$$
\hat{\mathbf{v}}_{\mathbf{u}_{k}}=\underset{\mathbf{c} \in \mathscr{C}}{\arg \min }\left\|\hat{\mathbf{R}}_{k}^{-1} \mathbf{v}_{\mathbf{r}_{k}}-\mathbf{v}_{\mathbf{c}}\right\|^{2},
$$

${ }^{1}$ Note that for constellations with rotational symmetry, more than one constellation point will correspond to the same Stokes vector; e.g., the PMQAM modulation format has a four-fold rotational symmetry, therefore four distinct constellation points $\mathbf{c}$ will correspond to the same Stokes vector $\mathbf{s}_{\mathbf{c}}$.

${ }^{2}$ The matrices $\boldsymbol{\lambda}_{2}$ and $\boldsymbol{\lambda}_{3}$ are not shown since they do not influence $R(\phi, \boldsymbol{\alpha})$ in (32). using the inverted estimate of $\mathbf{R}_{k}$. The estimate of the channel matrix $\hat{\mathbf{R}}_{k}$ is updated analogously to (18) as

$$
\hat{\mathbf{R}}_{k+1}=R\left(\hat{\phi}_{k}, \hat{\boldsymbol{\alpha}}_{k}\right) \hat{\mathbf{R}}_{k}
$$

where, analogously with (21)-(24) and (30),

$$
\begin{gathered}
\hat{\phi}_{k}=2 \mu_{\phi}\left(\hat{\mathbf{R}}_{k}^{-1} \mathbf{r}_{k}-\hat{\mathbf{u}}_{k}\right)^{\mathrm{T}} \hat{\mathbf{R}}_{k}^{-1} \boldsymbol{\lambda}_{1} \mathbf{r}_{k} \\
\hat{\boldsymbol{\alpha}}_{k}=-2 \mu_{\boldsymbol{\alpha}}\left(\hat{\mathbf{R}}_{k}^{-1} \mathbf{r}_{k}-\hat{\mathbf{u}}_{k}\right)^{\mathrm{T}} \hat{\mathbf{R}}_{k}^{-1} \overrightarrow{\boldsymbol{\rho}} \mathbf{r}_{k} .
\end{gathered}
$$

In the above description, only four DOFs, i.e., the scalars $\phi, \alpha_{1}, \alpha_{2}$, and $\alpha_{3}$ corresponding to $\lambda_{1}, \boldsymbol{\rho}_{1}, \boldsymbol{\rho}_{2}$, and $\boldsymbol{\rho}_{3}$, respectively, of the matrix $R(\phi, \boldsymbol{\alpha})$ were used, which correspond to the carrier phase and the SOP drift. The other two DOFs, i.e., the scalars corresponding to $\boldsymbol{\lambda}_{2}, \boldsymbol{\lambda}_{3}$ in (32), can be used to correct certain transmitter and/or receiver hardware imperfections, which cannot be done using Jones or Stokes formalisms, such as $90^{\circ} \mathrm{I} / \mathrm{Q}$ error or the time skew between I and Q [29].

The algorithm presented in this section is fully equivalent to the one in Section III-A and will have the same performance, but not to the one in Section III-B. The latter may have a different performance in terms of polarization tracking and a separate solution to mitigate the phase noise is required since it will not be covered by $\hat{\mathbf{M}}_{k}$. The polarization-tracking performance is degraded by the suboptimal error function (28), but at the same time, the performance may increase due to the four-fold reduction of the number of constellation points $\mathbf{s}_{\mathbf{c}}$ in the Stokes space for PM-QAM modulation formats, which increases the minimum Euclidean distance.

\section{RESULTS}

We evaluated the achievable performance of the proposed recovery algorithm numerically. The details of the simulation setup are described in Section IV-A, whereas in Sections IV-B to IV-E various performance metrics of the algorithm are evaluated.

\section{A. Simulation Setup}

The considered modulation formats are PS-QPSK, PMQPSK, PM-16-QAM, PM-64-QAM, and PM-256-QAM at a symbol rate of $28 \mathrm{Gbaud}$. The performance is quantified by counting the number of erroneous (4D) symbols to obtain the symbol error rate (SER) at the receiver for various setups in the presence of laser phase noise, SOP drift, and additive white Gaussian noise (AWGN). The latter is quantified through the signal-to-noise (SNR) ratio defined as $\mathrm{SNR}=E_{s} / N_{0}$. The four-fold phase ambiguity of QAM constellations is resolved using coherent differential coding [30, Sec. 2.6.1] employed independently in each polarization. At $\mathrm{SER}=10^{-3}$, coherent differential coding induces an SNR penalty of $\sim 0.44 \mathrm{~dB}$ for PS/PM-QPSK and decreases to $\sim 0.03 \mathrm{~dB}$ for PM-256-QAM. Note that the presented results when only AWGN is considered (for comparison reasons) still imply differential coding. These curves can be regarded as performance bounds, since these are the results that would be achieved with perfect phase and SOP tracking. The presented results are obtained using the Jones description of the algorithm from Section III-A. 
Table I

ALGORITHM PARAMETERS, ACHIEVABLE PERFORMANCE, AND HARDWARE COMPLEXITY

\begin{tabular}{|c|c|c|c|c|c|c|c|c|c|c|}
\hline & & $\begin{array}{l}\text { Algorithm } \\
\text { parameters }\end{array}$ & $\begin{array}{l}\text { Max. tol. } \\
\Delta p \cdot T\end{array}$ & $\begin{array}{l}\text { Max. tol. } \Delta p \\
\text { at } 28 \text { Gbaud }\end{array}$ & $\begin{array}{l}\text { Max. tol. } \\
\Delta \nu \cdot T\end{array}$ & $\begin{array}{l}\text { Max. tol. } \Delta \nu \\
\text { at } 28 \text { Gbaud }\end{array}$ & Adds. & Mults. & Comps. & $\begin{array}{l}\text { Memory } \\
\text { units }\end{array}$ \\
\hline \multirow{3}{*}{ PS-QPSK } & Kabsch & $\begin{array}{l}N_{\mathrm{Kab}}=31 \\
N_{\mathrm{BPS}}=13\end{array}$ & $0.46 \cdot 10^{-4}$ & $1.29 \mathrm{MHz}$ & $0.92 \cdot 10^{-4}$ & $2.58 \mathrm{MHz}$ & 63 & 69 & 7 & 0.5 \\
\hline & PS-CMA+BPS & $P_{\mathrm{BPS}}=32$ & $0.33 \cdot 10^{-4}$ & $0.93 \mathrm{MHz}$ & $6.80 \cdot 10^{-4}$ & $19.04 \mathrm{MHz}$ & 846 & 474 & 267 & 856 \\
\hline & Proposed alg. & $\begin{array}{l}\mu_{\mathrm{CMA}}=0.04 / E_{s}^{2} \\
c=27\end{array}$ & $3.50 \cdot 10^{-4}$ & $9.8 \mathrm{MHz}$ & $11.6 \cdot 10^{-4}$ & $32.48 \mathrm{MHz}$ & 145 & 201 & 7 & 8 \\
\hline \multirow{4}{*}{ PM-QPSK } & Kabsch & $N_{\mathrm{Kab}}=16$ & $0.28 \cdot 10^{-4}$ & $0.78 \mathrm{MHz}$ & $0.81 \cdot 10^{-4}$ & $2.27 \mathrm{MHz}$ & 80 & 91 & 4 & 1 \\
\hline & & $N_{\mathrm{BPS}}=19$ & & & & & & & & \\
\hline & $\mathrm{CMA}+\mathrm{BPS}$ & $P_{\mathrm{BPS}}=32$ & $0.37 \cdot 10^{-4}$ & $1.04 \mathrm{MHz}$ & $8.65 \cdot 10^{-4}$ & $24.22 \mathrm{MHz}$ & 2040 & 896 & 304 & 1264 \\
\hline & Proposed alg. & $\begin{array}{l}\mu_{\mathrm{CMA}}=0.16 / E_{s}{ }^{2} \\
c=64\end{array}$ & $1.61 \cdot 10^{-4}$ & $4.51 \mathrm{MHz}$ & $9.18 \cdot 10^{-4}$ & $25.70 \mathrm{MHz}$ & 145 & 201 & 4 & 8 \\
\hline \multirow{4}{*}{ PM-16-QAM } & Kabsch & $N_{\mathrm{Kab}}=16$ & $0.57 \cdot 10^{-5}$ & $159.6 \mathrm{kHz}$ & $0.16 \cdot 10^{-4}$ & $0.45 \mathrm{MHz}$ & 80 & 91 & 12 & 1 \\
\hline & & $N_{\mathrm{BPS}}=19$ & & & & & & & & \\
\hline & MMA+BPS & $P_{\mathrm{BPS}}=32$ & $0.14 \cdot 10^{-5}$ & $37.8 \mathrm{kHz}$ & $1.67 \cdot 10^{-4}$ & $4.68 \mathrm{MHz}$ & 2044 & 896 & 840 & 1264 \\
\hline & Proposed alg. & $\begin{array}{l}\mu_{\mathrm{MMA}}=0.04 / E_{s}{ }^{2} \\
c=400\end{array}$ & $3.14 \cdot 10^{-5}$ & $879.2 \mathrm{kHz}$ & $1.39 \cdot 10^{-4}$ & $3.89 \mathrm{MHz}$ & 145 & 201 & 12 & 8 \\
\hline \multirow{4}{*}{ PM-64-QAM } & Kabsch & $N_{\mathrm{Kab}}=16$ & $1.28 \cdot 10^{-6}$ & $35.8 \mathrm{kHz}$ & $0.35 \cdot 10^{-5}$ & $98.0 \mathrm{kHz}$ & 80 & 91 & 28 & 1 \\
\hline & & $N_{\mathrm{BPS}}=19$ & & & & & & & & \\
\hline & MMA+BPS & $P_{\mathrm{BPS}}=64$ & $0.11 \cdot 10^{-6}$ & $3.1 \mathrm{kHz}$ & $3.87 \cdot 10^{-5}$ & $1083.6 \mathrm{kHz}$ & 4040 & 1728 & 3806 & 2480 \\
\hline & Proposed alg. & $\begin{array}{l}\mu_{\mathrm{MMA}}=0.035 / E_{s}{ }^{2} \\
c=2352\end{array}$ & $6.79 \cdot 10^{-6}$ & $190.1 \mathrm{kHz}$ & $2.96 \cdot 10^{-5}$ & $828.8 \mathrm{kHz}$ & 145 & 201 & 28 & 8 \\
\hline \multirow{4}{*}{ PM-256-QAM } & Kabsch & $N_{\mathrm{Kab}}=16$ & $0.30 \cdot 10^{-6}$ & $8.4 \mathrm{kHz}$ & $0.83 \cdot 10^{-6}$ & $23.2 \mathrm{kHz}$ & 80 & 91 & 60 & 1 \\
\hline & & $N_{\mathrm{BPS}}=19$ & & & & & & & & \\
\hline & MMA+BPS & $P_{\mathrm{BPS}}=64$ & $8.42 \cdot 10^{-9}$ & $0.2 \mathrm{kHz}$ & $7.88 \cdot 10^{-6}$ & $220.6 \mathrm{kHz}$ & 4086 & 1728 & 8058 & 2480 \\
\hline & Proposed alg. & $\begin{array}{l}\mu_{\mathrm{MMA}}=0.017 / E_{s}{ }^{2} \\
c=6084\end{array}$ & $1.75 \cdot 10^{-6}$ & $49.0 \mathrm{kHz}$ & $7.40 \cdot 10^{-6}$ & $207.2 \mathrm{kHz}$ & 145 & 201 & 60 & 8 \\
\hline
\end{tabular}

Adds.=additions, mults.=multiplications, comps.=comparisons (see Sec. V).

The proposed algorithm was implemented such that both (22) and (24) were calculated for every $k$, i.e., $P=1$. The tracking step size $\mu_{\phi}$ and $\mu_{\boldsymbol{\alpha}}$ were chosen for each set of system parameters according to the heuristically obtained relations

$$
\begin{aligned}
& \mu_{\phi}=\frac{\sqrt{\Delta \nu T c}}{E_{s}}, \\
& \mu_{\boldsymbol{\alpha}}=\frac{\sqrt{\Delta p T c}}{E_{s}},
\end{aligned}
$$

where $c$ is a constant given in Table I, which depends on the modulation format. The constant $c$ was optimized to ensure the best performance in the steady-state regime at SER $=10^{-3}$. In some applications, the linewidth parameters of the optical link may be unknown at the receiver, and therefore it is impossible to accurately compute $\mu_{\phi}$ and $\mu_{\boldsymbol{\alpha}}$. To overcome this problem, the $\Delta p$ and $\Delta \nu$ parameters should be overestimated to be on the safe side. Typically, overestimating these parameters does not lead to considerable degradation in the performance.

For comparison, results obtained by the Kabsch algorithm [12] and combinations of the (PS-)CMA/MMA [2], [6], [7] and BPS [11] algorithms are presented. The Kabsch algorithm operates simultaneously on both polarizations in a decision-directed block-wise ${ }^{3}$ fashion, using a rectangular

\footnotetext{
${ }^{3}$ The algorithm can be modified to be used with a sliding window, and such a modification may possibly increase the performance, as well as the complexity. No such modification yet exists in the literature, to our knowledge.
}

filter of size equal to $N_{\mathrm{Kab}}=16$ [12], except for PS-QPSK, where it was set to $N_{\mathrm{Kab}}=31$ since we observed better results with a longer block size. The BPS algorithm uses a slidingwindow technique in each polarization independently since a relative phase offset between the two polarization may occur. The length of the window was set to $N_{\mathrm{BPS}}=19$ [11], except for PS-QPSK, where for the same considerations as above the filter length was set to $N_{\mathrm{BPS}}=13$. The number of test phases of the BPS algorithm was set to $P_{\mathrm{BPS}}=32$ for PS-QPSK, PM-QPSK, PM-16-QAM and $P_{\mathrm{BPS}}=64$ for PM-64-QAM and PM-256-QAM [11]. The convergence parameter of the (PS-)CMA/MMA algorithm $\mu_{\mathrm{CMA}}$ was optimized to tolerate the most polarization noise and the chosen values are listed in Table I.

In Sections IV-B to IV-D, the SOP and phase tracking capabilities of the algorithms are evaluated and therefore, in order to accurately quantify the tracking performance and avoid misconvergence of the algorithms, it is assumed that the algorithms have converged to the true channel matrix $\mathbf{H}_{0}$, i.e., we set $\hat{\mathbf{H}}_{0}=\mathbf{H}_{0}$. Section IV-E presents results on the convergence rate of the algorithms, where the channel matrix $\mathbf{H}_{0}$ is unknown to the receiver. The achieved results are shown in Fig. 3, where each row corresponds to one of Sections IV-BIV-E and each column corresponds to a modulation format. We omitted results obtained for PS-QPSK and PM-QPSK in the figure for space reasons, and these are summarized in Table I. 

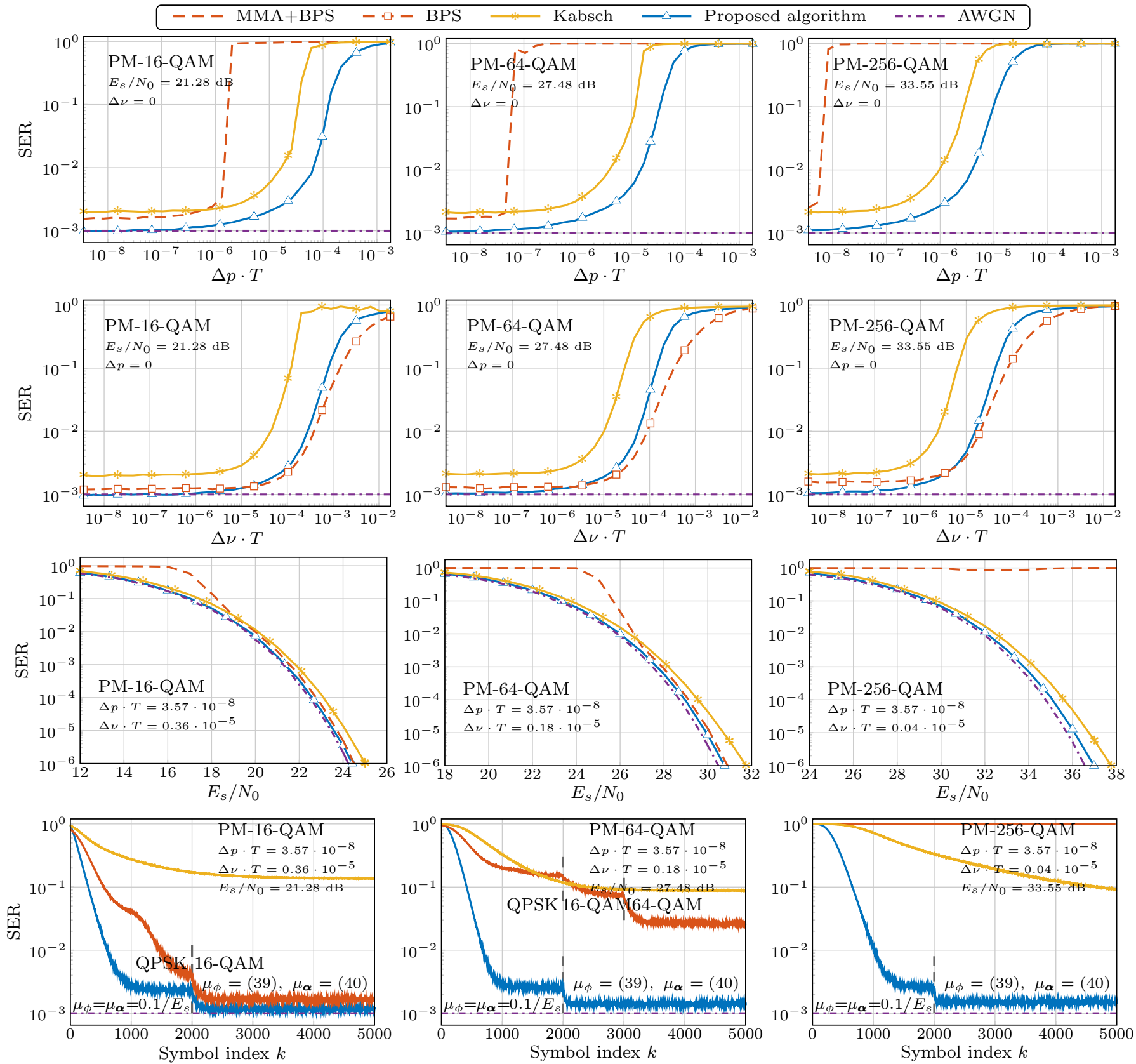

Figure 3. The achievable performance of the three tracking schemes for PM-16-QAM, PM-64-QAM, and PM-256-QAM is shown. Each column corresponds to a modulation format, whereas the rows present different performance metrics. The polarization-noise tolerance is shown in the first row by plotting SER versus $\Delta p \cdot T$. The tolerance to phase noise is plotted on the second row, where $\Delta \nu \cdot T$ is varied, whereas the noise sensitivity is shown in the third row by varying the SNR. The convergence rate is compared on the fourth row, where the SER is plotted versus the symbol index $k$.

\section{B. Polarization-Noise Tolerance}

In this section, the ability to track time-varying SOP drift is evaluated. To measure the polarization sensitivity, the polarization linewidth $\Delta p$ is varied while keeping the other parameters fixed. The SNR is set such that SER $=10^{-3}$ is achieved in an AWGN scenario and the accumulated laser linewidth is $\Delta \nu=0$.

The results of the simulation are shown in the top row of Fig. 3, where the SER is plotted versus the polarization linewidth times the symbol time. As can be seen, the proposed algorithm offers the best tolerance to SOP drift at any $\Delta p \cdot T$ for all modulation formats. High tolerance to SOP drift enables the use of high-order modulation formats even on rapidly varying channels, such as aerial fibers. Both competitor algorithms show error floors higher than the proposed algorithm, possibly due to the block-wise operation of the Kabsch algorithm and finite number of test phases of the BPS algorithm ${ }^{4}$, respectively. Perhaps the performance of both competitor algorithms could be improved if the block size/tracking step/number of test phases would be optimized for each set of system parameters.

${ }^{4}$ Even though phase noise is not present in this simulation setup, the BPS is still employed since the output of the MMA may have an absolute and/or relative phase offset introduced by the MMA and/or by the drift of the SOP. 
However, this is outside the scope of the paper. The performance of the MMA-based algorithms degrades significantly as the size of the constellation increases, providing the worst performance for PM-256-QAM.

Table I summarizes the maximum-tolerable polarization linewidth times the symbol time such that the receiver requires an extra $1 \mathrm{~dB}$ SNR to achieve $\mathrm{SER}=10^{-3}$ compared to the case without polarization noise. As can be seen in Table I, the proposed algorithm performs the best in all scenarios, being able to tolerate up to nine times more polarization noise.

\section{Phase-Noise Tolerance}

Another important feature of the considered tracking algorithms is the tolerance to phase noise. The phase-noise sensitivity is evaluated by varying $\Delta \nu$ and keeping the rest of the parameters fixed. The SNR is set such that a $\mathrm{SER}=10^{-3}$ is achieved in an AWGN scenario and $\Delta p=0$.

Fig. 3, second row, shows results obtained by the evaluated algorithms, where the SER is plotted versus the accumulated laser linewidth times the symbol time. The proposed algorithm has similar performance compared to BPS, which is known to have one of the best phase-noise sensitivities for QAM modulation formats [31].

Table I summarizes the maximum tolerable laser linewidth times the symbol time for an extra $1 \mathrm{~dB}$ SNR to achieve $\mathrm{SER}=10^{-3}$ compared to the case without laser noise. As can be seen in Table I, the proposed algorithm performs the best in all scenarios, except for PM-16-QAM, PM-64-QAM, and PM-256-QAM, where it has a slightly worse phase-noise sensitivity, being able to tolerate laser linewidths up to 32 MHz.

\section{Additive-Noise Sensitivity}

Fig. 3, third row, shows the SER as a function of SNR for fixed $\Delta p$ and $\Delta \nu$ shown in the figure. The Kabsch algorithm has the biggest penalty compared to the AWGN scenario, up $1.2 \mathrm{~dB}$ for PM-256-QAM, while the proposed algorithm has the smallest penalty for all three modulation formats with a maximum of $0.4 \mathrm{~dB}$ for PM-256-QAM. The performance of the MMA+BPS combination is in between the two other algorithms, but is the worst at low SNR.

\section{E. Convergence Rate}

An important quality of non-data aided algorithms is the convergence rate based on blind data. To compare the convergence rates of the three algorithms, additional numerical simulations have been performed, in which the initial channel matrix $\mathbf{H}_{0}$ was not known by the algorithms and generated by the method described in Section II. Note that the channel matrix was dynamic and updated according to (14). In order to compare the algorithms in a fair manner, the initial estimates of the channel matrix were set to be the identity, i.e., $\hat{\mathbf{H}}_{0}=\mathbf{I}_{2}$ or $\mathbf{I}_{4}$ for the Kabsch algorithm.

The convergence rates for the different modulation formats are shown in Fig. 3, bottom row, where the SER evolution is plotted versus the symbol index $k$. The results were obtained by averaging over $4 \cdot 10^{5}$ realizations, where in each realization the initial channel matrix was randomly generated. To improve the convergence rate of the MMA algorithm, the training regime has been split into several stages with increasing numbers of target radii from QPSK to the final constellation [32], as is shown in the figures. To further improve the convergence rate, the error function of the MMA can be modified as in [5] at the cost of a weakened tolerance to phase noise. The performance of the Kabsch algorithm could be improved by splitting the training regime into different stages with different window lengths $N_{\mathrm{Kab}}$, but it is outside the scope of this work. However, it is worth mentioning that the Kabsch algorithm has been proposed in [12] to be used in conjunction with training sequences such that the convergence stage is avoided by estimating the channel from the training sequence. The convergence rate of the proposed algorithm is improved by dividing the training regime into two stages, each with different tracking parameters $\mu_{\phi}, \mu_{\boldsymbol{\alpha}}$. The first stage, $k=1, \ldots, 2000$, uses the same value for both step sizes $\mu_{\phi}=\mu_{\boldsymbol{\alpha}}=0.1 / E_{s}$, which is bigger than the ones used in the second stage, $k>2000$, where the values were obtained using (39) and (40). It was observed that high step sizes give faster convergence but less accurate tracking in steady state.

Fig. 3, bottom row, shows that the proposed algorithm provides the fastest convergence compared to the other two algorithms and it approaches the AWGN scenario the closest. In the considered scenarios, the convergence takes up to 2500 symbols, depending on the constellation, corresponding to $\sim 100$ ps.

The convergence rate of the three algorithms can be improved by parallelization [33], where the computations are performed for a number of different initial matrices in parallel and the best candidate is chosen at a later stage.

\section{HARDWARE COMPLEXITY}

The assessment of the hardware complexity of the three considered algorithms is done by comparing the number of required real $^{5}$ additions, multiplications, comparisons, and memory units. Although this approach of quantifying complexity does not accurately measure the algorithm's efficiency and is a rough approximation, it is a starting point.

For pedagogical reasons, in (18), (29), and (36), the estimate of the channel matrix is computed (instead of the estimate of the inverse), which is then inverted and used throughout the rest of the algorithm. The inversion step is unnecessary and can be skipped by estimating the inverse of the channel matrix already in (18), (29), and (36) by negating $\hat{\phi}_{k}, \hat{\boldsymbol{\alpha}}_{k}$, thus reducing the required number of operations. In addition, calculations that occurs multiple times in the decoding procedure, e.g., $\hat{\mathbf{H}}_{k}^{-1} \mathbf{r}_{k}$ in (17), (22), (24), are computed only once. This holds for all three algorithms.

In Table I, the obtained results corresponding to one processed 4D symbol are listed. In this evaluation, the required number of operations was minimized by using memory units

\footnotetext{
${ }^{5}$ The complex multiplications have been converted to real operations such that a complex multiplication requires four real multiplications and two real summations.
} 
instead. This choice highly affects the BPS algorithm, where instead of recomputing previously done calculations for every processed symbol, the values are stored in the memory. Therefore, the results are different from the ones presented in [34], where no memory was used.

As can be seen in Table I, the Kabsch algorithm ${ }^{6}$ has the lowest complexity since it operates in a block-wise fashion processing the entire block at a time. This reduces the complexity significantly, which depends on the block length, i.e., a longer block will require less computing power per symbol, but also degrades the performance since it assumes that the channel does not change during the block. The (PS-)CMA/MMA+BPS combination requires the highest computational effort and it increases with the constellation size and the size of the sliding-window over which the BPS algorithm operates. The complexity of the proposed algorithm was evaluated using the Jones description, which is the same with respect to different modulation formats and it is in between the other two algorithms. The overall complexity of the algorithm can be reduced if the SOP tracking is not updated at every symbol in (18), as discussed in Section III-A. This is a reasonable approach since the SOP does not drift as fast as the phase noise and it can be considered constant over a limited observation time in some scenarios. In this case, if $\hat{\boldsymbol{\alpha}}_{k}$ is computed every $P$ symbols and $\hat{\phi}_{k}$ (which compensates for the phase noise) is updated at every symbol, the number of required operations is reduced from 346 (Table I) to $203+143 / P$. Of course in this scenario the performance will degrade, thus resulting in less tolerable polarization noise, but still sufficient for most installed fiber links.

\section{Algorithmic SUMmary}

This section provides an easily implementable form of the proposed algorithm without requiring knowledge about the details of the derivations.

The algorithm is summarized in Algorithm 1, where for ease of notation we denote $\mathbf{G}_{k}=\hat{\mathbf{H}}_{k}^{-1}$ to be the inverted estimate of the channel matrix at time $k$. The algorithm receives as inputs the received symbol $\mathbf{r}_{k}$, the previous inverse estimate of the channel matrix $\mathbf{G}_{k}$, and the symbol index $k$, and outputs the decided symbol $\hat{\mathbf{u}}_{k}$ and the updated matrix $\mathbf{G}_{k}$. The step sizes, which should be precomputed, are higher during convergence $(k \leq 2000)$ than tracking $(k>2000)$. In the tracking stage, they are computed based on the laser linewidth $\Delta \nu$ and the polarization linewidth $\Delta p$, which should be overestimated if they are unknown to the receiver. The Pauli matrices $\vec{\sigma}$ are given in (10), $\mathscr{C}$ is the set of constellation points, $E_{s}$ is the average symbol energy (4), $T$ is the symbol time, and $H(\cdot)$ is computed according to (15).

To decrease the computational effort, for slowly-varying channels, the update of $\mathbf{G}_{k}$ can be done less frequently than for every received symbol. Moreover, since the SOP drift varies slower than the phase noise, the SOP update of $\mathbf{G}_{k}$ can be

\footnotetext{
${ }^{6}$ We considered the Golub-Reinsch method [35, p. 493] for singular value decomposition required by the algorithm.
}

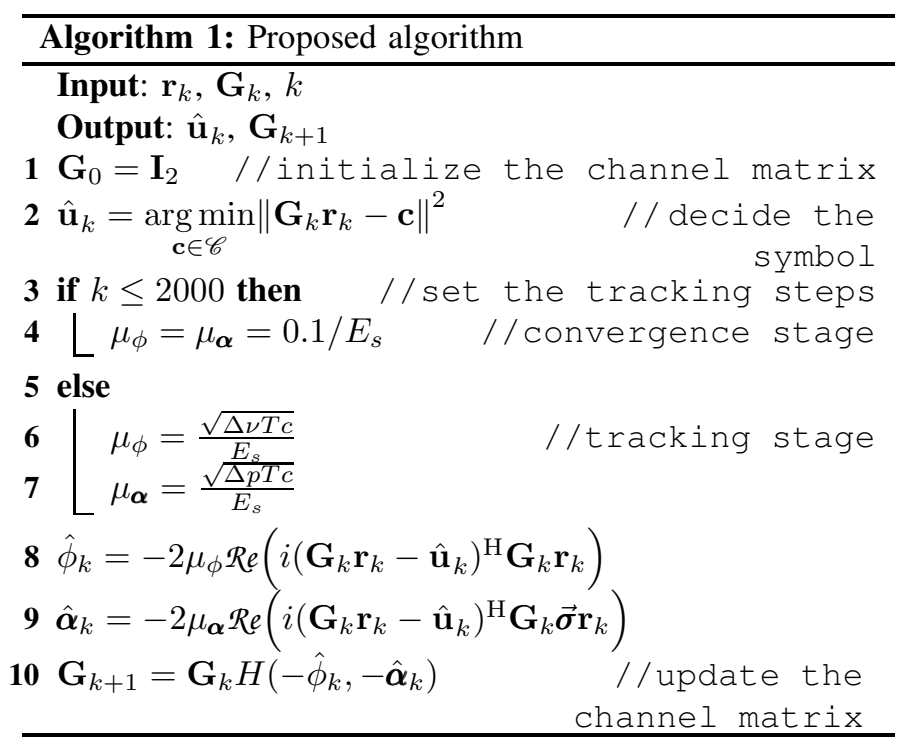

done less often by not calculating $\hat{\boldsymbol{\alpha}}_{k}$ in line 9 at each iteration but setting it to $\hat{\boldsymbol{\alpha}}_{k}=[0,0,0]^{\mathrm{T}}$ instead.

The description shown in Algorithm 1 uses the Jones formalism. This can be interchanged with the Stokes or real 4D formalisms by replacing the Jones matrix $\mathbf{G}_{k}$ with $\hat{\mathbf{M}}_{k}^{-1}$ (Section III-B) or $\hat{\mathbf{R}}_{k}^{-1}$ (Section III-C), and $\hat{\phi}_{k}, \hat{\boldsymbol{\alpha}}_{k}$ should be calculated using (30) or (37)-(38), respectively. In this case, the Jones vectors $\mathbf{r}_{k}, \hat{\mathbf{u}}_{k}$ become Stokes vectors or real $4 \mathrm{D}$ vectors. The Jones description of the algorithm is fully equivalent with the real 4D description and they achieve the same performance. However, the latter involves more computations since multiplying two $4 \times 4$ real matrices requires more operations than multiplying two $2 \times 2$ complex matrices. Nevertheless, the real 4D description can be modified to account for hardware imperfections. On the other hand, the Stokes description is immune to absolute phase shifts and only tracks the SOP, requiring a separate solution for absolute phase tracking. This can be beneficial in some situations since fast oscillations of the phase noise will not affect the algorithm, and therefore the SOP tracking. Performance-wise, the Stokes description will behave differently from the other two as it relies on a suboptimal error function (28), but at the same time, the performance may increase due to the four-fold reduction of the number of constellation points $\mathbf{s}_{\mathbf{c}}$ in the Stokes space for PM-QAM modulation formats, which increases the minimum Euclidean distance.

\section{DISCUSSION AND CONCLUSIONS}

We have proposed a model-based algorithm to jointly track random polarization and phase drifts. The algorithm uses the gradient descent optimization algorithm in a decisiondirected architecture processing one symbol at a time. The achievable performance of the algorithm was evaluated by means of numerical simulations and compared to state-of-theart algorithms. Results show the effectiveness of the proposed algorithm, having a fast convergence rate and an excellent tolerance to phase noise and dynamic drifts of the polarization, in particular when using high-order modulation formats. At 
similar or better performance, the computational complexity of the proposed algorithm is considerably lower compared to CMA/MMA+BPS, whereas compared to the Kabsch algorithm, our algorithm provides at least a five-fold performance improvement at the price of doubling the complexity. The increased phase- and polarization-noise tolerance for high-order modulation formats at low complexity makes the algorithm a strong candidate for future optical systems.

The proposed algorithm is tested in a somewhat idealized scenario assuming that the polarization-mode dispersion is negligible, which is not the case for long-haul transmission. The performance of the proposed algorithm will be affected if polarization-mode dispersion is present, which limits the applicability of the algorithm. However, the algorithm can be applied after a separate equalization stage as in [36] that compensates for polarization-mode dispersion neglecting the SOP, after which the SOP is corrected by the proposed algorithm.

\section{APPENDIX}

In this appendix, the gradient of the error function (19) with respect to $\phi, \boldsymbol{\alpha}$ is derived as

$$
\begin{aligned}
\nabla_{\boldsymbol{\alpha}} e_{k}=\nabla_{\boldsymbol{\alpha}}\left\|\left(H(\phi, \boldsymbol{\alpha}) \hat{\mathbf{H}}_{k}\right)^{-1} \mathbf{r}_{k}-\hat{\mathbf{u}}_{k}\right\|^{2} \\
=\operatorname{Re}\left(2\left(\left(H(\phi, \boldsymbol{\alpha}) \hat{\mathbf{H}}_{k}\right)^{-1} \mathbf{r}_{k}-\hat{\mathbf{u}}_{k}\right)^{\mathrm{H}}\right. \\
\left.\cdot \nabla_{\boldsymbol{\alpha}}\left(\left(H(\phi, \boldsymbol{\alpha}) \hat{\mathbf{H}}_{k}\right)^{-1} \mathbf{r}_{k}-\hat{\mathbf{u}}_{k}\right)\right) \\
=2 \operatorname{Re}\left(\left(\hat{\mathbf{H}}_{k}^{-1} H(-\phi,-\boldsymbol{\alpha}) \mathbf{r}_{k}-\hat{\mathbf{u}}_{k}\right)^{\mathrm{H}} \hat{\mathbf{H}}_{k}^{-1}\right. \\
\left.\cdot \nabla_{\boldsymbol{\alpha}} H(-\phi,-\boldsymbol{\alpha}) \mathbf{r}_{k}\right),
\end{aligned}
$$

where (42) follows because, for any $\mathbf{y} \in \mathbb{C}^{n}$ and $\mathbf{x} \in \mathbb{R}^{m}$, $\nabla_{\mathbf{x}}\|\mathbf{y}\|^{2}=\nabla_{\mathbf{x}}\left(\mathbf{y}^{\mathrm{H}} \mathbf{y}\right)=2 \operatorname{Re}\left(\mathbf{y}^{\mathrm{H}} \nabla_{\mathbf{x}} \mathbf{y}\right)$. The partial derivatives of $H(-\phi,-\boldsymbol{\alpha})=e^{i \phi} J(-\boldsymbol{\alpha})$ are, from (11) and (15),

$$
\begin{aligned}
& \frac{\partial H(-\phi,-\boldsymbol{\alpha})}{\partial \phi}=i e^{i \phi} J(-\boldsymbol{\alpha}), \\
& \frac{\partial H(-\phi,-\boldsymbol{\alpha})}{\partial \alpha_{i}}=e^{i \phi} \frac{\partial J(-\boldsymbol{\alpha})}{\partial \alpha_{i}} \\
&=e^{i \phi} \frac{\partial\left(\mathbf{I}_{2} \cos \theta+i \mathbf{a} \cdot \overrightarrow{\boldsymbol{\sigma}} \sin \theta\right)}{\partial \alpha_{i}} \\
&=e^{i \phi}\left(-\mathbf{I}_{2} a_{i} \sin \theta+i\left(\frac{\boldsymbol{\sigma}_{i}}{\theta}-\frac{a_{i}}{\theta} \mathbf{a} \cdot \overrightarrow{\boldsymbol{\sigma}}\right) \sin \theta\right. \\
&\left.+i \mathbf{a} \cdot \overrightarrow{\boldsymbol{\sigma}} a_{i} \cos \theta\right)
\end{aligned}
$$

where (47) follows because $\partial \theta / \partial \alpha_{i}=\alpha_{i} /\|\boldsymbol{\alpha}\|=a_{i}$.

Evaluating (44) and (47) at $\phi=0, \boldsymbol{\alpha}=[0,0,0]^{\mathrm{T}}$ results in

$$
\frac{\partial H(-\phi,-\boldsymbol{\alpha})}{\partial \phi}=i \mathbf{I}_{2}, \quad \frac{\partial H(-\phi,-\boldsymbol{\alpha})}{\partial \alpha_{i}}=i \boldsymbol{\sigma}_{i},
$$

as $\theta \rightarrow 0$ for any direction $\mathbf{a}$. The gradient of the error function can be obtained by substituting (48) and $H(0,0)=\mathbf{I}_{2}$ in (43), which is then substituted in (21) and (23) to obtain (22) and (24), respectively.

\section{REFERENCES}

[1] R. Kim, M. O’Sullivan, Kuang-Tsan Wu, Han Sun, A. Awadalla, D. Krause, and C. Laperle, "Performance of dual-polarization QPSK for optical transport systems," Journal of Lightwave Technology, vol. 27, no. 16, pp. 3546-3559, Aug. 2009.

[2] S. J. Savory, "Digital coherent optical receivers: algorithms and subsystems," IEEE Journal of Selected Topics in Quantum Electronics, vol. 16, no. 5, pp. 1164-1179, Sept.-Oct. 2010.

[3] C. B. Czegledi, M. Karlsson, E. Agrell, and P. Johannisson, "Polarization drift channel model for coherent fibre-optic systems," Nature Scientific Reports, vol. 6, no. 21217, Feb. 2016.

[4] D. N. Godard, "Self-recovering equalization and carrier tracking in two-dimensional data communication systems," IEEE Transactions on Communications, vol. 28, no. 11, pp. 1867-1875, Nov. 1980.

[5] J. Yang, J.-J. Werner, and G. A. Dumont, "The multimodulus blind equalization and its generalized algorithms," IEEE Journal on Selected Areas in Communications, vol. 20, no. 5, pp. 997-1015, Jun. 2002.

[6] H. Louchet, K. Kuzmin, and A. Richter, "Improved DSP algorithms for coherent 16-QAM transmission," in Proc. European Conference on Optical Communication (ECOC), Brussels, Belgium, Sept. 2008, p. Tu.1.E.6.

[7] P. Johannisson, M. Sjödin, M. Karlsson, H. Wymeersch, E. Agrell, and P. A. Andrekson, "Modified constant modulus algorithm for polarizationswitched QPSK," Optics Express, vol. 19, no. 8, pp. 7734-7741, Apr. 2011.

[8] M. Visintin, G. Bosco, P. Poggiolini, and F. Forghieri, "Adaptive digital equalization in optical coherent receivers with Stokes-space update algorithm," Journal of Lightwave Technology, vol. 32, no. 24, pp. 47594767, Dec. 2014.

[9] N. J. Muga and A. N. Pinto, "Adaptive 3-D Stokes space-based polarization demultiplexing algorithm," Journal of Lightwave Technology, vol. 32, no. 19, pp. 3290-3298, Oct. 2014.

[10] A. Viterbi and A. Viterbi, "Nonlinear estimation of PSK-modulated carrier phase with application to burst digital transmission," IEEE Transactions on Information Theory, vol. 29, no. 4, pp. 543-551, Jul. 1983.

[11] T. Pfau, S. Hoffmann, and R. Noé, "Hardware-efficient coherent digital receiver concept with feedforward carrier recovery for $M$-QAM constellations," Journal of Lightwave Technology, vol. 27, no. 8, pp. 989-999, Apr. 2009.

[12] H. Louchet, K. Kuzmin, and A. Richter, "Joint carrier-phase and polarization rotation recovery for arbitrary signal constellations," IEEE Photonics Technology Letters, vol. 26, no. 9, pp. 922-924, May 2014.

[13] D. A. Morero, M. A. Castrillón, A. Aguirre, M. R. Hueda, and O. E. Agazzi, "Design trade-offs and challenges in practical coherent optical transceiver implementations," Journal of Lightwave Technology, vol. 34, no. 1, pp. 121-136, Jan. 2016.

[14] J. N. Damask, Polarization Optics in Telecommunications. New York, NY: Springer, 2005.

[15] E. Agrell and M. Karlsson, "Power-efficient modulation formats in coherent transmission systems," Journal of Lightwave Technology, vol. 27, no. 22, pp. 5115-5126, Nov. 2009.

[16] M. Tur, B. Moslehi, and J. Goodman, "Theory of laser phase noise in recirculating fiber-optic delay lines," Journal of Lightwave Technology, vol. 3, no. 1, pp. 20-31, Feb. 1985.

[17] R. Bellman, Introduction to Matrix Analysis. New York, NY: McGrawHill, 1960.

[18] J. P. Gordon and H. Kogelnik, "PMD fundamentals: polarization mode dispersion in optical fibers," Proceedings of the National Academy of Sciences of the United States of America, vol. 97, no. 9, pp. 4541-4550, Apr. 2000.

[19] M. Karlsson, C. B. Czegledi, and E. Agrell, "Coherent transmission channels as 4d rotations," in Proc. Signal Processing in Photonic Communication (SPPCom), Boston, MA, Jul. 2015, p. SpM3E.2.

[20] M. Karlsson, "Four-dimensional rotations in coherent optical communications," Journal of Lightwave Technology, vol. 32, no. 6, pp. 12461257, Mar. 2014

[21] H. Sun and K.-T. Wu, "A novel dispersion and PMD tolerant clock phase detector for coherent transmission systems," in Proc. Optical Fiber Communication Conference (OFC), Los Angeles, CA, Mar. 2011, p. OMJ4. 
[22] T. Nakagawa, K. Ishihara, T. Kobayashi, R. Kudo, M. Matsui, Y. Takatori, and M. Mizoguchi, "Wide-range and fast-tracking frequency offset estimator for optical coherent receivers," in Proc. European Conference on Optical Communication (ECOC), Torino, Italy, Sept. 2010, p. We.7.A.2.

[23] S. Boyd and L. Vandenberghe, Convex Optimization. New York, NY: Cambridge University Press, 2004.

[24] K. Ogaki, M. Nakada, Y. Nagao, and K. Nishijima, "Fluctuation differences in the principal states of polarization in aerial and buried cables," in Proc. Optical Fiber Communication Conference (OFC), Atlanta, GA, Mar. 2003, p. MF13.

[25] P. Krummrich, E.-D. Schmidt, W. Weiershausen, and A. Mattheus, "Field trial results on statistics of fast polarization changes in long haul WDM transmission systems," in Proc. Optical Fiber Communication Conference $(O F C)$, Anaheim, CA, Mar. 2005, p. OThT6.

[26] C. B. Czegledi, E. Agrell, and M. Karlsson, "Symbol-by-symbol joint polarization and phase tracking in coherent receivers," in Proc. Optical Fiber Communication Conference (OFC), Los Angeles, CA, Mar. 2015, p. W1E.3.

[27] S. Betti, F. Curti, G. De Marchis, and E. Iannone, "A novel multilevel coherent optical system: 4-quadrature signaling," Journal of Lightwave Technology, vol. 9, no. 4, pp. 514-523, Apr. 1991.

[28] R. Cusani, E. Iannone, A. M. Salonico, and M. Todaro, "An efficient multilevel coherent optical system: M-4Q-QAM," Journal of Lightwave Technology, vol. 10, no. 6, pp. 777-786, Jun. 1992.

[29] D. E. Crivelli, M. R. Hueda, H. S. Carrer, M. del Barco, R. R. López, P. Gianni, J. Finochietto, N. Swenson, P. Voois, and O. E. Agazzi, "Architecture of a single-chip $50 \mathrm{~Gb} / \mathrm{s}$ DP-QPSK/BPSK transceiver with electronic dispersion compensation for coherent optical channels," IEEE Transactions on Circuits and Systems I: Regular Papers, vol. 61, no. 4, pp. 1012-1025, Apr. 2014.

[30] M. Seimetz, High-Order Modulation for Optical Fiber Transmission. Heidelberg, Germany: Springer, 2009.

[31] X. Zhou, "Efficient clock and carrier recovery algorithms for singlecarrier coherent optical systems: a systematic review on challenges and recent progress," IEEE Signal Processing Magazine, vol. 31, no. 2, pp. 35-45, Mar. 2014

[32] F. P. Guiomar, S. B. Amado, A. Carena, G. Bosco, A. Nespola, A. Teixeira, and A. N. Pinto, "Fully-blind linear and nonlinear equalization for 100G PM-64QAM optical systems," Journal of Lightwave Technology, vol. 33, no. 7, pp. 1265-1274, Apr. 2015.

[33] P. Johannisson, H. Wymeersch, M. Sjödin, A. S. Tan, E. Agrell, P. A. Andrekson, and M. Karlsson, "Convergence comparison of the CMA and ICA for blind polarization demultiplexing," Journal of Optical Communications and Networking, vol. 3, no. 6, pp. 493-501, Jun. 2011.

[34] J. H. Ke, K. P. Zhong, Y. Gao, J. C. Cartledge, A. S. Karar, and M. A. Rezania, "Linewidth-tolerant and low-complexity two-stage carrier phase estimation for dual-polarization 16-QAM coherent optical fiber communications," Journal of Lightwave Technology, vol. 30, no. 24, pp. 3987-3992, Dec. 2012.

[35] G. H. Golub and C. F. Van Loan, Matrix Computations, 4th ed Baltimore, MD: Johns Hopkins Univ. Press, 2013.

[36] F. Buchali, H. Bülow, K. Schuh, and W. Idler, "4D-CMA: enabling separation of channel compensation and polarization demultiplex," in Proc. Optical Fiber Communication Conference (OFC), Los Angeles, CA, Mar. 2015, p. Th2A.15. 